\title{
Characterising 3D Objects by Shape and Topology
}

\author{
Stina Svensson ${ }^{1}$, Carlo Arcelli², and Gabriella Sanniti di Baja ${ }^{2}$ \\ 1 Centre for Image Analysis \\ Swedish University of Agricultural Sciences, Uppsala, Sweden \\ stina@cb.uu.se \\ 2 Istituto di Cibernetica \\ National Research Council of Italy, Pozzuoli (Napoli), Italy \\ $\{$ car,gsdb\}@imagm.cib.na.cnr.it
}

\begin{abstract}
Information on the shape of an object can be combined with information on the shape of the complement of the object, in order to describe objects having complex shape. We present a method for decomposing and characterising the convex deficiencies of an object, i.e., the regions obtained by subtracting the object from its convex hull, into parts corresponding to cavities, tunnels, and concavities of the object. The method makes use of the detection of watersheds in a distance image.
\end{abstract}

Keywords: Distance transform, watershed segmentation, topological erosion, volume image.

\section{Introduction}

The description of objects having complex shape, but that are not easily decomposable into meaningful simple parts, can be achieved if also the shape of the complement of the object, the background, is investigated. In fact, object and background play dual roles, and concavities of the object can be described as convexities of the background. The analysis of the entire background can be rather time consuming and, for this reason, only the voxels of the background that are embedded in concavities of the object should be taken into account. Therefore, it is convenient to compute the convex hull of the object, since in this way the portion of the background to be investigated can be limited to the convex deficiencies, i.e., the difference between the convex hull and the object, [1]. A bounding box could be used instead of the convex hull to save computation time. However, the use of a bounding box would only limit the size of the portion of the background to be investigated, but would not provide useful hints to achieve object's description. In fact, the difference between the bounding box and the object seldom originates components that can be in faithful correspondence with the perceived convex deficiencies.

Topological features involve both the object and its complement and, as such, are particularly useful for the description of non-intuitively decomposable complex objects. Topological features of objects in 3D images are the connected 

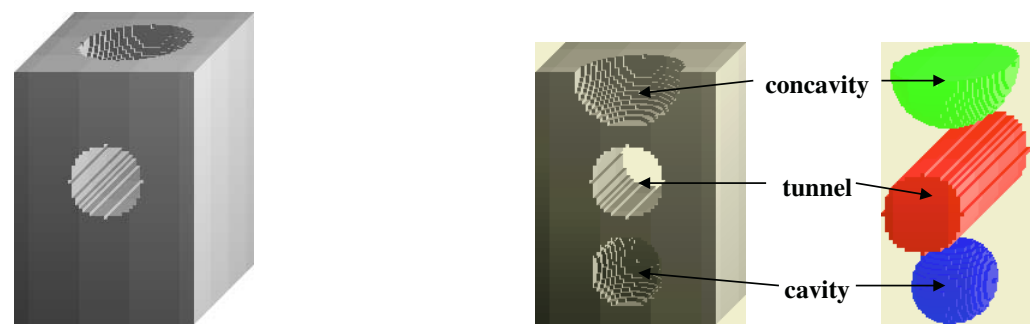

Fig. 1. Object with one concavity, one tunnel, and one cavity, left. A cross section of the object and both topological and shape features, right.

components of the object, called object components, and for each object component, the connected components of the background that are completely enclosed by the object component, also called cavities, and tunnels. A tunnel exists whenever a component of the background is interpenetrating the object. Besides topological features, also some shape features are of interest for the description of non-intuitively decomposable complex objects. For example, this is the case for concavities of the object that can be interpreted as convexities or protrusions of the background. Concavities of the object, as well as tunnels and cavities can be identified by computing the convex hull and by analysing the convex deficiencies. A simple case is given in Fig. 1. There, a brick-shaped object consisting of one connected component is shown. The object includes a concavity, visible on top of the object, a tunnel, crossing the object in the middle, and a cavity on the bottom of the object, visible only in the cross section of the object.

While the number of object components and the number of cavities are easy to compute by means of local operators, tunnels are more difficult to identify and only recently some contributions have appeared dealing with this subject.

In [2], an algorithm was presented to close tunnels, called holes in that article. The purpose of that algorithm was actually that of detecting tunnels (especially in nearly thin objects, as it is clear by looking at the examples shown in the article) in order to suppress them (or to suppress only those considered as less significant, based on the size of the tunnels). In this respect, the algorithm works nicely as it identifies a closure located in the middle of each tunnel. However, our purpose is to count, represent and describe complex tunnels in object characterised by any thickness. In this sense, the algorithm 2] is not adequate. In fact, for a complex tunnel consisting of crossing branches it is not possible to know a priori how many distinct closures will be identified, since this depends on the length of the different branches.

More recently, a method to detect tunnels (and cavities) and to represent them by linear structures (and single voxels) has been introduced in 3 . The method is based on the topological erosion of the convex deficiencies of the object. The number of crossing points (or better clusters of crossing points) found within the linear representation of the tunnel is used to estimate tunnel complexity, and the number of end points accounts for the number of exits of the 
tunnel. Moreover, information on the maximal thickness of the tunnel is given in terms of the number of iterations of topological erosion necessary to generate the linear representation.

In this paper, we perform the analysis of concavities, tunnels and cavities, still using the convex deficiencies of the object. In particular, we here face the problem of decomposing convex deficiencies, corresponding to a number of concavities and tunnels merged into a unique component, into the constituting entities. To this aim, we use a combination of constrained distance transformation and watershed segmentation. Once the convex deficiencies have been decomposed into individual entities, the method in [3] can be applied to extract from each entity its representation.

\section{Preliminaries}

We consider volume images consisting of object and background. We treat the object as 26-connected, i.e., two object voxels are adjacent if they share a face, an edge, or a vertex, and the background as 6-connected, i.e., two background voxels are adjacent if they share a face. For an object voxel $v$, we denote $N^{26}(v)$, the set of voxels from the immediate neighbourhood of $v$ including all face, edge, and vertex neighbours of $v$, and $N^{18}(v)$, the set of voxels including all face and edge neighbours of $v$.

An object component is a set of voxels for which each pair of voxels, $u$ and $v$ can be connected by a path, $u=w_{0}, w_{1}, \ldots, w_{n}=v$, within the object and such that $w_{i+1} \in N^{26}\left(w_{i}\right), i=0, \ldots, n-1$. For simplicity, in this paper we will consider a volume image including a single object component. In case of more than one object component, connected component labelling (performed by using, e.g., the algorithm in 4]) is preliminarily performed, so as to work on each object component individually.

The convex hull of an object is the smallest convex set containing that object. Different, equivalent, definitions for a convex set $S$ exist, e.g., a set is convex when for all points $P, Q \in S$ the straight line connecting $P$ and $Q$ is also in $\mathrm{S}$. Defining and finding the convex hull of a discrete object is not trivial, [5]. Often an approximation of the convex hull, e.g., a covering polyhedron, is adequate. In this paper, we use the method described in [6] to build a covering polyhedron by repeatedly applying concavity filling operators. Though using $3 \times 3 \times 3$ operators, the method actually derives and uses information from a $5 \times 5 \times 5$ neighbourhood of each voxel, to establish whether the voxel is located in a planar region. Thus, the resulting approximation of the convex hull is quite good, as the covering polyhedron is characterised by a number of up to 90 faces. An even larger number of faces could be obtained by deriving and using information from a larger neighbourhood. However, for our purpose this is not necessary, as the increased accuracy does not affect the result enough to justify the increase in computational cost.

The convex deficiencies of an object are obtained by computing the difference between the covering polyhedron and the object. In what follows, we denote the 

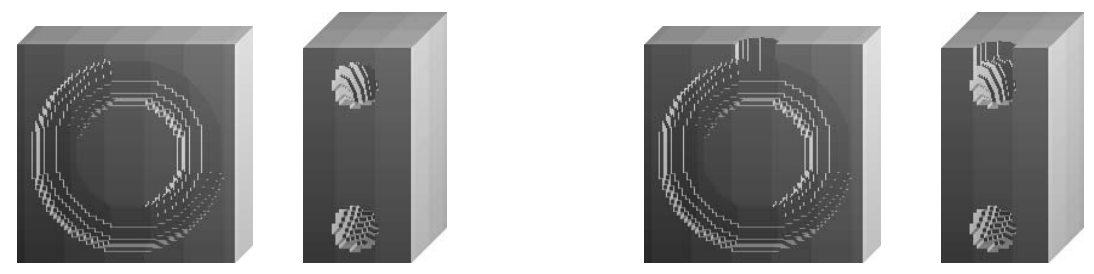

Fig. 2. Two cross sections of a box with a cavity shaped as a torus, left, and of a box with a concavity including a torus, right.

convex deficiencies by CDs. For each CD we call cap each connected component of voxels belonging to the $\mathrm{CD}$ and having at least a face-neighbour in the complement of the covering polyhedron.

An object has a tunnel if there exists a closed connected path in the object which cannot be deformed to a single voxel (for details, see 7). A tunnel is identified by a CD having more than one cap (two caps for a simple tunnel, more than two caps for tunnels consisting of many branches). An object has a cavity if a background component is fully enclosed in the object. A cavity is identified by a CD having no cap at all. An object has a concavity, whenever a CD including a single cap is found. We note that using the number of caps to establish the nature of a CD allows us to be consistent also in presence of otherwise ambiguous cases. For example, see Fig. 2 left, where both the definition of cavity (i.e., a background component fully enclosed by the object) and the definition of tunnel (i.e., a background region such that there exists a closed connected path that cannot be deformed to a single voxel) apply. By using the number of caps, the $\mathrm{CD}$ is classified as a cavity. Analogously for the example shown in Fig. 2, right, the CD is classified as a concavity, though part of it is clearly shaped as a tunnel.

An object voxel $v$ is simple if the object including $v$ is homotopic to the object obtained after $v$ has been assigned to the background, 8]. This means that the number of object components, the number of tunnels, and the number of cavities is the same, independently of whether $v$ is in the object or in the background. A decision on whether $v$ is simple or not can be taken based on the local neighbourhood configuration of $v$, 910. The voxel $v$ is simple if the number of object components in $N^{26}(v)$ is one and the number of background components, having $v$ as a face neighbour, in $N^{18}(v)$ is also one.

Topological erosion of the object is a process that assigns simple voxels to the background. The process terminates when no more object voxels are simple.

Distance between voxels or sets of voxels in an image can be represented by means of a distance transform, [1]. In a distance transform, each voxel in the object is assigned a value corresponding to the distance to its closest voxel in a reference set, which is often the background. A good approximation to the Euclidean distance, i.e., a distance that is stable under rotation, can be obtained by taking the distance between two voxels as the length of the minimal path between the voxels, where each step in face direction is weighted 3, each step in edge direction is weighted 4 , and each step in vertex direction is weighted 5, [12. 
We will use this distance function through this paper. In case the reference set with respect to which the distance is computed is a subset of the object, instead of the background, the distance transform is said to be constrained, [13].

\section{Decomposing the Convex Deficiencies of an Object}

We first briefly summarise the method introduced in [3] to associate a representation to cavities and tunnels, because this constitutes the final part of the procedure described in this paper. In [3], once the covering polyhedron is achieved and the difference to the object is computed, the CDs are identified using a connected component labelling algorithm, [4], to assign an identity label to each component. Among the CDs, cavities could be easily distinguished as the CDs having no face neighbour in the background. However, all CDs, including those corresponding to cavities, undergo the topological erosion, done to detect the relative representations, because these structures are easier to manage than the CDs, and carry enough information for shape description. Topological erosion of the CDs is accomplished by removing simple voxels having no face or edge neighbours in the complement of the covering polyhedron. To guide the erosion through successive, more and more internal, voxels of the CDs, the constrained distance transform of the CDs (called DT, for short) is computed, where the reference set from which to derive distance information is the original object. Using DT also allows us to associate with each CD, information concerning its maximal thickness, given by the maximal distance label found within the DT. The resulting representation will consist, for each cavity, in an isolated voxel having no face neighbours in the complement of the covering polyhedron, and, for each tunnel, in a linear structure where a number of voxels have edge or vertex neighbours in the complement of the covering polyhedron (the voxels are as many as the exits of the tunnel). If CDs corresponding to concavities are also found, an isolated voxel having edge or vertex neighbours in the complement of the covering polyhedron is found for each connected set of concavities.

A limit of the above method is that whenever a CD corresponds to a combination of more than a single entity, e.g., a number of concavities or a number of tunnels and concavities, the obtained representation only accounts for one entity, namely the thickest concavity, if the CD is in correspondence with a combination of concavities, or the tunnels, in case of a combination of tunnels and concavities. As an elucidative example, consider the object in Fig. 3 There, a solid brick-shaped object is shown, from which a number of cylinders and (parts of) balls have been removed to create tunnels and concavities. Though eight entities are perceived (two tunnels and six concavities), only four CDs are found: one simple concavity; one simple tunnel; one component consisting of the combination of one tunnel and three concavities; and one component consisting of the combination of two concavities. The corresponding representations are shown in Fig. 4 where we note that the structures corresponding to the combination of concavities and tunnels and to the combination of concavities, account only for the tunnel and for one concavity, respectively. 

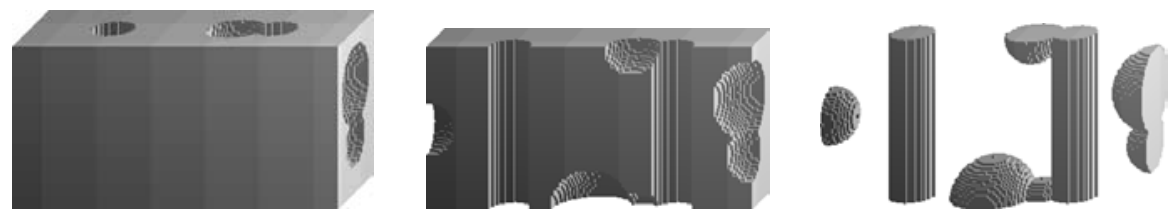

Fig. 3. From left to right: object, a cross section and the convex deficiencies.

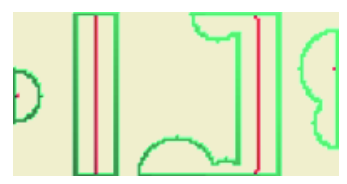

Fig. 4. Representation of the convex deficiencies for the object in Fig. 3, framed by a cross section of the borders of the CDs.

It is clear that to obtain correct representations by the method in [3], the CDs corresponding to combinations of concavities and combinations of tunnels and concavities should be preliminarily decomposed into their constituting entities. In this paper, we aim at achieving a decomposition of these CDs into parts corresponding to single tunnels and single concavities.

To decompose CDs corresponding to combinations of concavities or combinations of tunnels and concavities, we resort to watershed segmentation, 14,15. The concept of watershed is based on the idea of a "topographic" interpretation of a multi-valued image, e.g., a grey-level image or a distance transform where distance labels play the role of grey levels. The three spatial coordinates $x, y$, and $z$ of a voxel $v$, together with elevation of $v$, which is the grey-level of $v$, are used. This gives raise to an elevation model in terms of a hyper-surface. In this interpretation we have three types of voxels: voxels that are minima; voxels belonging to catchment basins; and voxels belonging to watersheds (crest lines). See Fig. [5] for the 2D case. Watersheds are found by "immersion". Imagine each minimum as pierced so that when immersing the hyper-surface into water, the catchment basins start to be filled. A watershed is built in correspondence with any voxel which is reached by water coming from two basins.

To identify the minima within the CDs, we compute the DT, where we use as reference set the complement of the covering polyhedron. The minima for the watershed segmentation are the voxels farthest from the reference set. Accordingly, they are detected as the maxima on the DT. In correspondence of each tunnel most of maxima will be placed midway with respect to the caps delimiting the tunnel. A problem to be solved is that the number of connected components of maxima generally exceeds the number of entities, so that an over-segmentation is likely to be obtained. Well known techniques to reduce this over-segmentation can be applied, e.g. , see [16. We do not discuss here these techniques, but concentrate on additional criteria we adopt to reduce over-segmentation. Specif- 


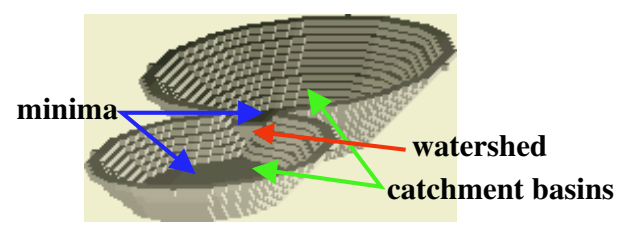

Fig. 5. Voxels involved in watershed computation.
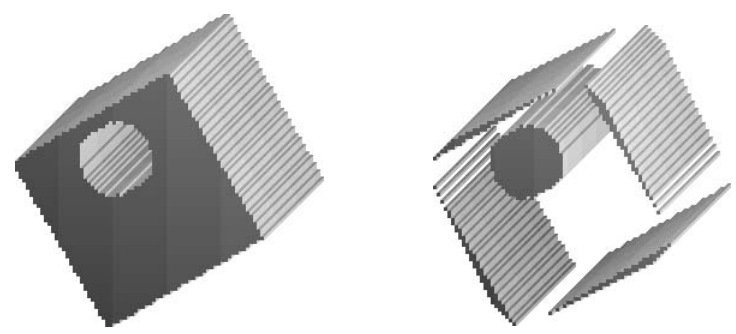

Fig. 6. Object, left, and its convex deficiencies, right. Only the convex deficiency corresponding to the tunnel is meaningful.

ically, our criteria involve the reduction of the number of components of maxima, merging of components of maxima, and merging of parts of the decomposition.

To reduce the number of components of maxima, we perform a small number of erosion/dilation operations. This results in smoothed CDs, since spurs and thin protrusions are removed. We note that erosion/dilation is also useful to avoid considering spurious CDs. In fact, when building the covering polyhedron, concavity filling changes the status of a number of voxels that are not really placed in concavities of the object, but are such that the planes passing through them and tangent to the object are not oriented according to permitted directions. Thus, CDs are likely to be identified even when no concavities are actually present in the object. In Fig. 6] a cube rotated $30^{\circ}$ in $z$-direction with respect to the upright position is shown together with the found CDs. It can be noted that besides the expected CD corresponding to the tunnel, also other four spurious CDs are detected. In fact, the faces of the cube are not oriented along directions permitted for the faces of the covering polyhedron. Thus, in correspondence with each face of the cube, concavity filling adds to the covering polyhedron all voxels understood as belonging to local concavities as far as a face of the covering polyhedron oriented along a permitted direction is obtained.

The tool used to merge components of maxima is active only for maxima found midway with respect to the caps delimiting each tunnel. To this aim, we need to compute closures in correspondence with tunnels and accomplish this task similarly to [2]. Since the DT is already available, we can use it to guide a topological erosion that removes simple voxels, starting from the voxels having minimal distance label and proceding inwards, until the closures are obtained. The process is illustrated in Fig. 7 where a brick-shaped object with 

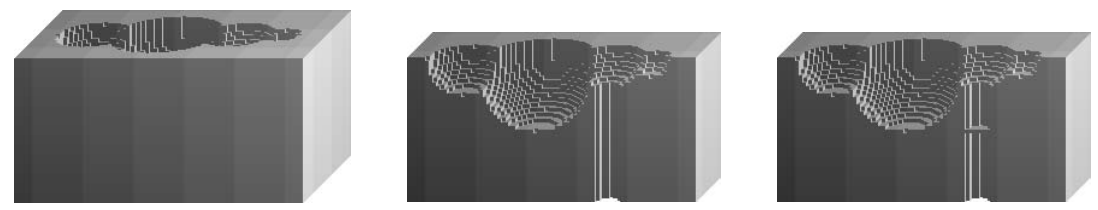

Fig. 7. Object with a combination of concavities and a tunnel, left, a cross section, middle, and the closure of the tunnel, right.
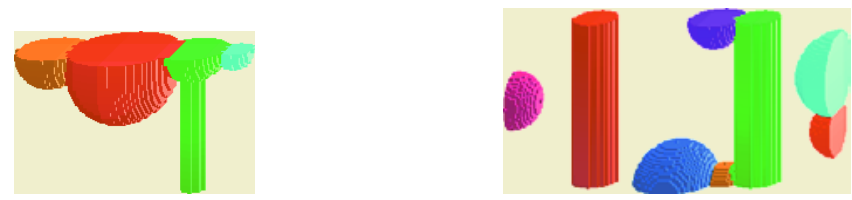

Fig. 8. Decomposition for the CDs of the object in Fig. 7 left, and in Fig. 3 right. (Fig. 8 is actually in colours. For a better understanding, please refer to the electronic version of the paper.)

a combination of concavities and a tunnel is shown. The closure of the tunnel is shown in Fig. 7 right.

Connected component labelling of the closures is accomplished. Then, we can ascribe the same identity label to all components of maxima found midway with respect to the caps delimiting the tunnels. These maxima are either included in a closure (and as such already have the same identity label as the closure they belong to), or are adjacent to it (and the identity label of the closure can be assigned to them). The latter case occurs when the length of the tunnel is expressed by an even number of voxels and, hence, the set of maxima is two-voxel thick.

Connected component labelling is, then, accomplished on the remaining maxima. This completes the process to identify the markers for the watershed segmentation. We use an algorithm for computing the watersheds which is basically an extension to deal with 3D images of the algorithm presented in [14].

The watershed decomposition for the CDs of the objects in Fig. 7 and in Fig. 3, are respectively shown in Fig. 8 to the left and the right.

As concerns merging among parts of the decomposition, we distinguish two cases respectively dealing with complex tunnels, and with tunnels or concavities with significant protrusions that have not been removed by erosion/dilation. For tunnels having complex shape and, hence, more than one branch, e.g., a Y-shaped tunnel, more than one closure can be found. As a consequence it may happen that, after the watershed segmentation, a branch of the tunnel is assigned more than one identity label. See Fig. 9. Merging of the parts identified within the tunnel can be easily accomplished. In fact, closures have been assigned identity labels that not only distinguish a closure from other closures, but also discriminate between components of maxima found in correspondence with a closure and all other components of maxima. The second merging case 

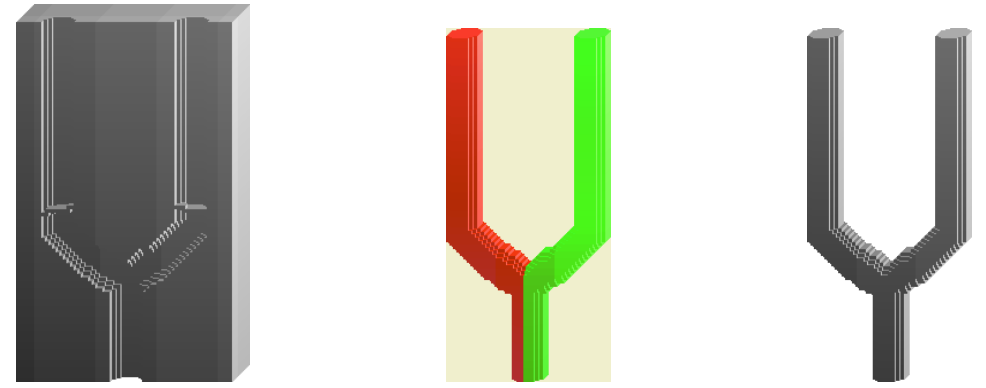

Fig. 9. A Y-shaped tunnel with the found two closures, left, watershed segmentation before merging, middle, and after merging, right.
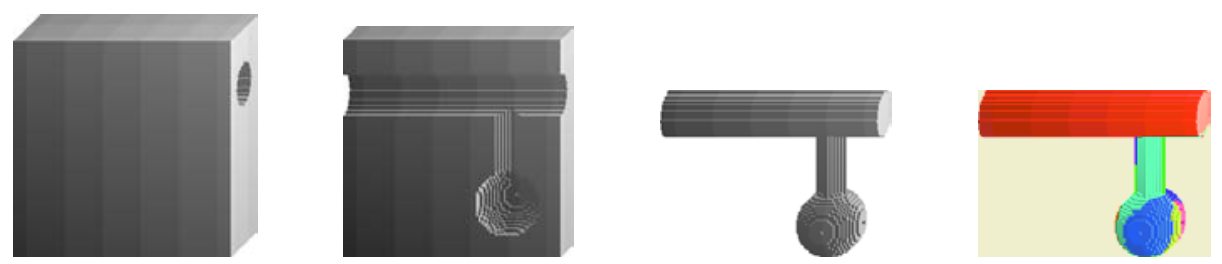

Fig. 10. From left to right: a tunnel with a significant protrusion, a cross section, its convex deficiencies, and watershed segmentation before merging. Result after merging is one tunnel part. (Fig. 10 is actually in colours. For a better understanding, please refer to the electronic version of the paper.)

regards entities with significant protrusions, see Fig. 10. When this is the case, maxima in the DT are found also in correspondence with the significant protrusions of the tunnel which will cause creation of parts of the decomposition, once watershed segmentation is implemented. Since according to our definition, all entities, except cavities, have at least one cap, we merge all adjacent parts without cap until a compound part with cap is achieved.

Once all parts of the decomposition have at least one cap, over-segmentation can be treated by well known techniques. Finally, the representations consisting in a linear structure for tunnels and in isolated voxels for concavities and cavities can be obtained by using the method described in [3].

\section{Conclusion}

We have characterised an object in a 3D binary image in terms of topology and shape by analysing the convex deficiencies of (an approximation of) the convex hull of the object. While the identification of cavities is a trivial problem, detection of tunnels and concavities is often tricky. Various techniques have been used, including distance transformation, connected component labelling, watershed segmentation and topological erosion. The method has given satisfactory results, when tested on a large set of artificial objects. No evidence is available 
yet of the effectiveness of the method on real images. We expect that new problems will arise that have not occurred with the artificial objects used so far. This will be a topic for future research.

\section{References}

1. Borgefors, G., Sanniti di Baja, G.: Analyzing nonconvex 2D and 3D patterns. Computer Vision and Image Understanding 63 (1996) 145-157

2. Aktouf, Z., Bertrand, G., Perroton, L.: A three-dimensional holes closing algorithm. Pattern Recognition Letters 23 (2002) 523-531

3. Svensson, S., Arcelli, C., Sanniti di Baja, G.: Finding cavities and tunnels in 3D complex objects. Proceedings of 12th International Conference on Image Analysis and Processing (ICIAP 2003), Mantova, Italy, IEEE CS (in press)

4. Thurfjell, L., Bengtsson, E., Nordin, B.: A new three-dimensional connected components labeling algorithm with simultaneous object feature extraction capability. CVGIP: Graphical Models and Image Processing 54 (1992) 357-364

5. Soille, P.: Morphological Image Analysis. Springer-Verlag (1999)

6. Borgefors, G., Nyström, I., Sanniti di Baja, G.: Computing covering polyhedra of non-convex objects. In: Proceedings of 5th British Machine Vision Conference, York, UK (1994) 275-284

7. Kong, T.Y.: A digital fundamental group. Computers \& Graphics 13 (1989) $159-166$

8. Kong, T.Y., Rosenfeld, A.: Digital topology: Introduction and survey. Computer Vision, Graphics, and Image Processing 48 (1989) 357-393

9. Saha, P.K., Chaudhuri, B.B.: Detection of 3-D simple points for topology preserving transformations with application to thinning. IEEE Transactions on Pattern Analysis and Machine Intelligence 16 (1994) 1028-1032

10. Bertrand, G., Malandain, G.: A new characterization of three-dimensional simple points. Pattern Recognition Letters 15 (1994) 169-175

11. Rosenfeld, A., Pfaltz, J.L.: Distance functions on digital pictures. Pattern Recognition 1 (1968) 33-61

12. Borgefors, G.: On digital distance transforms in three dimensions. Computer Vision and Image Understanding 64 (1996) 368-376

13. Piper, J., Granum, E.: Computing distance transformations in convex and nonconvex domains. Pattern Recognition 20 (1987) 599-615

14. Vincent, L., Soille, P.: Watersheds in digital spaces: An efficient algorithm based on immersion simulations. IEEE Transactions on Pattern Analysis and Machine Intelligence 13 (1991) 583-597

15. Beucher, S., Lantuejoul, C.: Use of watersheds in contour detection. In: International Workshop on image processing: Real-time edge and motion detection/estimation. (1979) Rennes, France.

16. Meyer, F.: An overview of morphological segmentation. International Journal of Pattern Recognition and Artificial Intelligence 15 (2001) 1089-1118 